\title{
Evaluation de la sensibilité des cépages autochtones Algériens au phylloxera (Daktulosphaira vitifoliae)
}

\author{
Salima Sebki, Kaddour El Heit ${ }^{\mathrm{a}}$, Abderazak Hamama, Saida Meghezzi, Ounissa Agouazi et Mohand Said Cherfaoui \\ Laboratoires des Ressources Naturelles: Viticulture/Arboriculture, Faculté des Sciences et des Sciences Agronomiques, \\ Université Mouloud Mammeri, Tizi-Ouzou 15.000, Algérie
}

\begin{abstract}
Résumé. Le phylloxera radicicole (Daktulosphaira vitifoliae) est l'un des facteurs majeurs qui a causé la régression des cépades locaux d'Algérie. Notre travail s'inscrit dans l'objectif d'évaluation la sensibilité de 16 cépages autochtones (vitis vinifera L. ssp. vinifera), de deux porte-greffes ( $\mathrm{SO}_{4}$ et Rupestris du Lot) et d'un individu de V. sylvestris à l'égard de cet insecte redoutable. La méthode de contamination appliquée est celle préconisé par Boubals (1966). Les bois récoltés des cépages à étudier ont été confectionnés en boutures à deux yeux et mise en pots contenant un substrat formé de (2/3) d'argile et (1/3) de grignon d'olive et conservées dans une serre tunnel contrôlée. Le test de sensibilité a été réalisé en prélevant sur les porte-greffes sensibles des champs de vignes mères, les feuilles couvertes de galles de phylloxera, puis enterrées dans les pots de boutures à tester. Cette opération d'inoculation a été réalisée en trois reprises. Deux semaines après la dernière inoculation, les observations faites sur racines nous renseignent sur le comportement de phylloxera radicicole au niveau du système racinaire. Les attaques de ces pucerons ont provoqué des lésions de gravité variable en fonction de chaque cépage, à l'exception des porte-greffes Rupestris du Lot et $\mathrm{SO}_{4}$ qui semblaient être résistants. En effet, le greffage sur ces espèces américaines reste le seul moyen fiable et efficace contre ces attaques.
\end{abstract}

\section{Introduction}

La vigne est l'espèce la plus cultivée dans le monde, vu son importance économique considérable. Le raisin est commercialisé comme raisin de table, jus de fruit et particulièrement pour la production de vins. Mais malheureusement, la vigne cultivée est sujette à de nombreuses maladies et ravageurs qui ont provoqué des dégâts souvent considérables et des pertes de récoltes, aussi en engendrant la disparition de nombreux cépages autochtones.

Comme c'est le cas en Algérie. Elle était très riche en biodiversité de la vigne, mais au cours du dernier siècle, plusieurs facteurs ont causé la régression des cépages mineurs, depuis l'introduction dans la viticulture traditionnelle des variétés du continent européen plus productives et mieux adaptées aux nouvelles techniques vinicoles. Cette régression est accentuée à la suite de la diffusion du phylloxera.

Le phylloxera a été identifiée par Bazille en 1863, il est l'ennemi le plus redoutable, originaire d'Amérique où il vivait sur les vignes sauvages qui ne semblaient pas en souffrir, envahit rapidement des vignobles dont il provoquait leur destruction [1]. Actuellement presque la totalité des vignobles du monde sont sujets à cet insecte [5].

Ce ravageur a également porté l'appellation scientifique Pemphigus vitifoliae, Phylloxera vastratris et Vitus vitifolii, avant son appellation actuelle de Dactylosphaera

\footnotetext{
a e-mail : kaddy60023@yahoo.fr
}

vitifoliae [6]. Il se manifeste sous ces deux formes, gallicole, sur les feuilles des vignes américaines et radicicole en s'attaquant surtout aux racines des vignes européennes. Cette dernière forme est la plus dangereuse et dévastatrice.

$\mathrm{Vu}$ qu'il a été toujours considéré comme l'une des plus dangereuses maladies du vignoble Algérien, notre étude s'est basée sur la contribution à évaluer la sensibilité des cépages de Vitis vinifera L. ssp. vinifera autochtones algériens au phylloxera radicicole, par rapport au portegreffe Rupestris du Lot, $\mathrm{SO}_{4}$ et un cultivar appartenant à Vitis vinifera ssp. sylvestris.

\section{Matériels et méthodes}

Douze cépages ont fait l'objet de notre travail : Ahmar Bou Amer, Aneb El Kadi, Muscat d'El Adda, Sbaa Tolba, Amellal, Ahchichane, Ghanez, Lakhdari, Toutrissine, Amghar, Ferrana blanc, Ferrana de mascara, Cherchali, Aberkane et Ain El Bouma.

Nous avons entrepris la récolte du bois au niveau de la collection régionale de cépages autochtones de Médéa, ainsi que les porte-greffes au champs de pieds mères à la ferme EAC 07 Hamza Mohamed de Blida. Le matériel végétal récolté est entreposé au froid à $4{ }^{\circ} \mathrm{C}$ pendant un mois, ensuite nous avons procédé à la multiplication végétative de notre matériel suivant le protocole classique de production de plants en pépinière.

Les boutures issues de la multiplication sont mises en serre dans des pots contenant $2 / 3$ de la terre riche en argile et $1 / 3$ de grignon d'olive pour assurer une bonne reprise

This is an Open Access article distributed under the terms of the Creative Commons Attribution License 4.0, which permits unrestricted use, distribution, and reproduction in any medium, provided the original work is properly cited. 

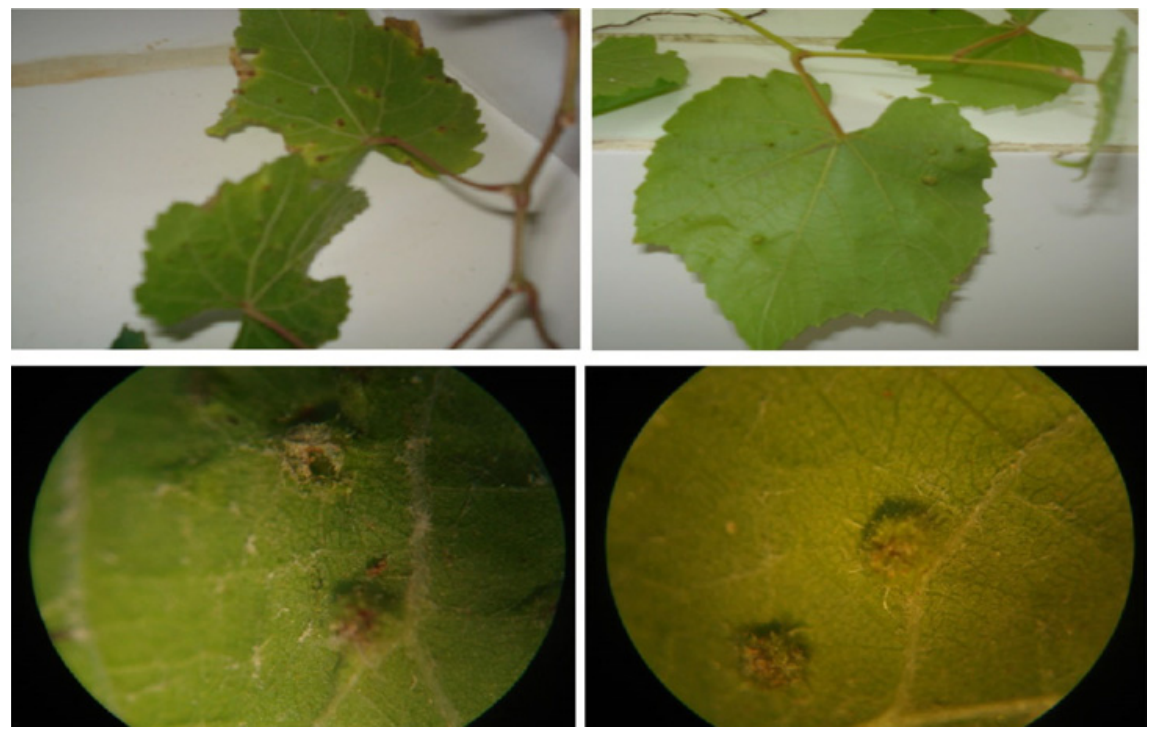

Figure 1. Apparition des galles phylloxériques au niveau des feuilles du porte-greffe $\mathrm{SO}_{4}$ et Rupestris du Lot.
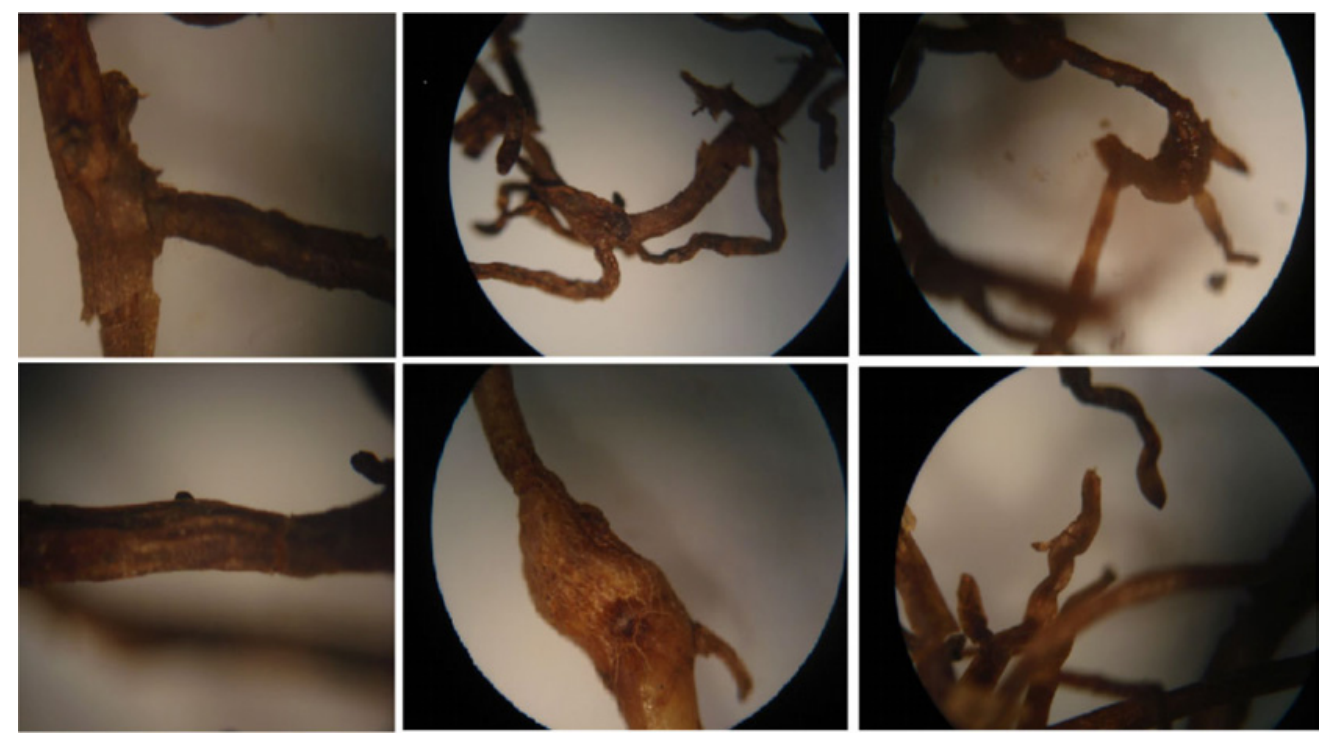

Figure 2. Tubérosités et nodosités sur les racines des cépages autochtones vues sous loupe binoculaire.

pour les plants et un milieux favorable au développement de l'insecte.

Le test a été réalisé selon le protocole décrit par Boubals (1966). Des feuilles couvertes de galles de phylloxera ont été prélevées sur le porte-greffe 41B (Chasselas $\times$ Vitisberlandiri) connu pour sa grande sensibilité au phylloxera gallicole [2], puis enterrés dans les pots des boutures à tester. Cette opération est réalisée en trois reprises intercalées de 45 jours.

A la fin de 1'automne, les plants ont été dépotés pour observer la présence ou l'absence des nodosités et des tubérosités développées sur le système racinaire. Les observations faites sur racines nous renseignent sur le comportement de phylloxera radicicole au niveau du système racinaire.

L'ensemble des résultats obtenus ont été soumis à des analyses de variance, on utilisant le logiciel STAT-BOX v. 6.4 .

\section{Résultats et discussion}

\subsection{Symptomatologie}

Après 15 jours de la dernière inoculation, nous avons remarqué que les plants des porte-greffes Rupestris du Lot et $\mathrm{SO}_{4}$ testés ont manifesté des symptômes caractéristiques du phylloxera.

L'infection s'est traduite par des galles à la face inferieure de leurs feuilles, couvrant la totalité de la surface foliaire, qui se trouvent parfois groupées et parfois éparpillées. Leur forme est sphériques d'une couleur verte pour les galles récemment formées et rouge pour celles qui sont en stade avancé [1] (Fig. 1).

Par contre, au niveau des racines des boutures des cépages autochtones testées, après dépotage, les observations effectuées nous confirment la présence des symptômes qui se manifestent par l'apparition des nodosités et des tubérosités. Ces dernières se traduisent 


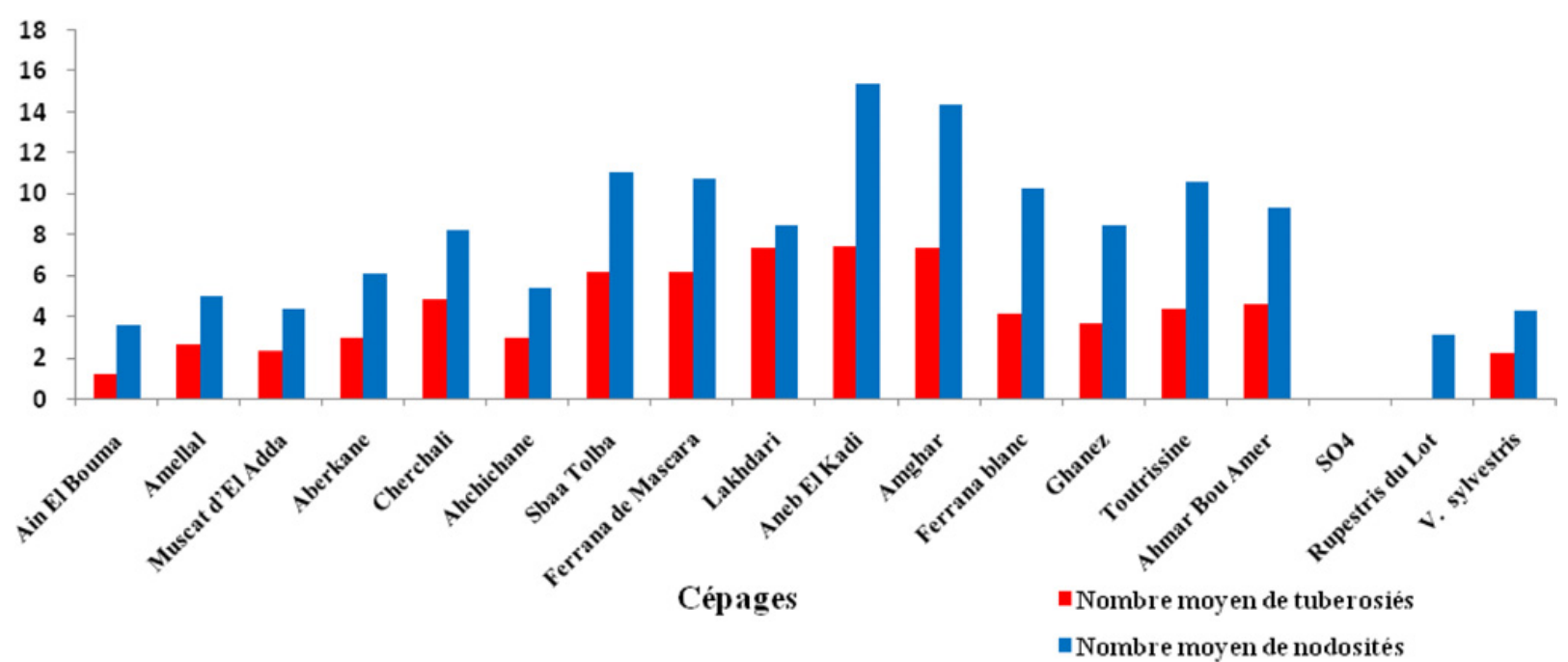

Figure 3. Moyenne du nombre de tubérosités et de nodosités observé pour chaque cépage.

Tableau 1. Analyse de la variance au seuil de $5 \%$ du nombre de tubérosités pour les cépages étudiées.

\begin{tabular}{|l|c|c|c|c|c|c|c|}
\hline & S.C.E & DDL & C.M. & TEST F & PROBA & E.T. & C.V. \\
\hline Var. Totale & 2993,558 & 305 & 9,815 & & & & \\
\hline Var. Facteur & 1618,029 & 17 & 95,178 & 19,928 & 0 & & \\
\hline Var. Résiduelle & 1375,529 & 288 & 4,776 & & & 2,185 & $55,59 \%$ \\
\hline
\end{tabular}

Tableau 2. Analyse de la variance au seuil de $5 \%$ du nombre de nodosités pour les cépages étudiées.

\begin{tabular}{|l|c|c|c|c|c|c|c|}
\hline & S.C.E & DDL & C.M. & TEST F & PROBA & E.T. & C.V. \\
\hline Var. Totale & 6643,559 & 305 & 21,782 & & & & \\
\hline Var. Facteur & 4730,853 & 17 & 278,286 & 41,902 & 0 & & \\
\hline Var. Résiduelle & 1912,706 & 288 & 6,641 & & & 2,577 & $33,32 \%$ \\
\hline
\end{tabular}

par des renflements, mais elles ne sont pas nombreuses puisque les racines ne sont pas bien développées. Par contre, la présence des nodosités a été plus significative, elles ont une apparence d'une tète d'oiseaux et se concentrent surtout au niveau des extrémités des radicelles (Fig. 2).

\section{2. Évaluation de phylloxera radicicole}

D'après la figure ci-dessous, nous remarquons que l'ensemble des génotypes semble être sensible aux attaques phylloxériques qui provoquent l'apparition de deux formes de lésions bien distinct; nodosités et tubérosités, mais à des degrés différents, surtout pour les cépages autochtones. Signalant que le nombre moyen le plus élevé de nodosités est enregistré pour Aneb El Kadi et Amghar et il se chiffre en moyenne à 15,41 et à 14,41 . De même pour le nombre de tubérosités qui s'élève à 7,47 et à 7,41 en moyenne. Ces données obtenues les classent comme étant les plus sensibles parmi les variétés étudiées. Par contre, cette même étude classe Ain El Bouma comme ayant le faible nombre de nodosités et de tubérosités avec en moyenne respectivement 3,64 et 1,23 . Les cépages restant ont une particularité médiane de sensibilité à l'insecte.
L'analyse de la variance (Tableaux 1 et 2 ) a montré une variation très hautement significative $(p=0)$ pour le nombre de tubérosités selon les variétés, de même pour le nombre de nodosités.

Nous avons remarqué que plus le nombre de nodosités augmentent le nombre de tubérosités est proportionnellement élevé. Cette situation serait dû aux plus grands nombre de radicelles développés par les jeunes plants connus pour être particulièrement très sensibles à manifester un plus grand nombre de tubérosités. De ce fait, cette approche s'avère logique.

Selon [7] le degré d'attaque de phylloxera radicicole diffère d'un cépage à l'autre, cela revient à la différence spécifique des gènes de ce matériel végétal. D'ailleurs pour le porte greffe Rupestris de Lot, nous avons enregistré une présence de nodosités plus élevées. En revanche le porte greffe $\mathrm{SO}_{4}$ présente une résistance remarquable à l'égard de cet insecte, aucune lésion n'est apparue. Sa résistance serait probablement due à son aptitude génétique.

Ce classement corrobore avec celui obtenu par les chercheurs cités précédemment. Ils ont classé la plupart des cépages cultivés de $V$. vinifera sativa dans la classe sensible à très sensibles. Tandis que dans la classification de très résistante à résistante, elle renferme la grande majorité les cépages américains. Quant aux variétés de 
l'espèce $V$. rotundifolia (le parent de $\mathrm{SO}_{4}$ ) ont été groupées dans la classe immune.

Des observations de sensibilité aux attaques phylloxériques ont été faites aussi par [4] lors de ces travaux effectués sur la caractérisation de cinq cépages autochtones; Avoukera, Arekak, Imerssasse, Avekan, Thiziri et de deux génotypes appartenant à $V$. vinifera L. ssp. silvestris. Les résultats obtenus avaient signalé que l'ensemble des variétés étudiées sont sensibles au phylloxera avec l'apparition des nodosités et des tubérosités au niveau des racines des plants testés, sauf pour le cépage autochtone Arekak chez qui les symptômes se sont manifestés uniquement par des nodosités.

Egalement [8] ont observé la sévérité du phylloxera radicicole sur des racines de deux cépages cultivés en France ( $V$. vinifera sativa). Ils ont noté sur le cépage de table Chasselas et du cuve Cabernet une moyenne du nombre de tubérosité qui est de 9,53;9,27 et une moyenne du nombre de nodosités égale à 32,$87 ; 41,13$. Or pour les porte-greffes $41 \mathrm{~B}$ et $333-\mathrm{EM}$ aucune lésion n'est apparue.

\section{Conclusion}

Après avoir évalué la sensibilité de quelques cépages autochtones d'Algérie au phylloxera radicicole avec le génotype lambrusque ainsi les deux porte-greffes $\mathrm{SO}_{4}$ et Rupestris du Lot, nous sommes arrivés à déduire que l'ensemble des cultivars étudiés, surtout les cultivars autochtones sont classés comme sensibles au phylloxera radicicole.

Ainsi, le greffage s'avère plus que nécessaire pour multiplier les cultivars de Vitis vinifera sur des porte-greffes résistants. C'est donc un moyen sûr pour se protéger de ce parasite du moins pour le moment, puisque de nouveaux biotypes de l'insecte de phylloxéra peuvent apparaitre.

La poursuite des recherches dans ce domaine s'avère intéressante afin d'approfondir les études et aussi pour bien surveiller continuellement, ce ravageur redoutable et assurer la pérennité et le patrimoine génétique des vignes surtout autochtones qui sont déjà menacées, ça serait aussi mieux d'élargir nos études afin d'évaluer la sensibilité d'autres maladies qui s'avèrent aussi menaçantes.

\section{Références}

[1] A. Reynier, 2005. Manuel de viticulture. 10éme édition. Lavoisier Tec \& Doc. Paris. 600 p.

[2] Alain Reynier, 2007. Manuel de viticulture. $9^{\circ}$ édition. Lavoisier Tec \& Doc. $\mathrm{N}^{\circ} 626$ (1). France. $554 \mathrm{p}$.

[3] D. Boubals, 1966. Etude de la distribution et des causes de la résistance au phylloxera radicicole chez les vitacées. Ann. Amél. Plantes (16). 145-185

[4] Hamama, 2008. Contribution à la caractérisation de cinq cépages autochtones (V.v ssp. Sativa) et deux lambrusques (V. v ssp. Silvestris) de la région de Timizart (W. Tizi Ouzou). Mémoire d'Ingénieur d'Etat en Sciences Agronomiques. Spécialité: production végétale. Option : cultures pérennes. Université Mouloud MAMMERI. Tizi Ouzou. 39-67

[5] J.P. Peros, 2003. Genec structure and variation in aggressiveness in European and Australian populations of the grapevine diabak fungus. Eur J. Plant Pathol. 109

[6] Perez Marin, 2007. Champignons in les parasites de la vigne, stratégies de protection raisonné. Editions. La vigne. DUNOD. $\mathrm{N}^{\circ}$ 5100. Paris. 193, 205

[7] R. Pouget et S.K.Kim, 1978. Etude méthodologique de la résistance au phylloxera : application à quelques croisements interspécifiques. In : Génétique et Amélioration de la vigne. II Symposium International sur l'Amélioration de la vigne. 189-197. INRA. Paris

[8] R. Ocete, M. Cantos, O. Failla, G. Lovicu, B. Biagini, S. Imazio, M. Lara, D. Maghradze et M. Angeles Lopez, 2011. Considérations on the European wild grappe vine (Vitis vinifera L. ssp. sylvestris (Gmelin) Hegi) and Phylloxera infestation. University of Sevilla. Spain. Vitis 50 (2). 97-98

[9] S. Divecchi, 2007. Caractérisation moléculaire des cépages autochtones italiens. Thèse de doctorat. Université de Florance. Italie. 132 p. 